\title{
Alterar limites e categorias de áreas protegidas é necessariamente ruim? Um estudo de caso em duas unidades de conservação estaduais da Amazônia brasileira
}

\section{Change the limits and categories of protected areas is necessarily bad? A study case in two state protected areas in the Brazilian Amazon}

Sérgio Borges - Professor do Departamento de Biologia da Universidade Federal do Amazonas (UFAM). Bolsista do Programa FIXAM (Edital 017/2014) da Fundação de Amparo à Pesquisa do Estado do Amazonas (FAPEAM). E mail: shborges9@g mail.com

Francisco Souza - Mestre em Gestão de Áreas Protegidas da Amazônia (MPGAP/INPA). Atua em conselhos gestores de várias unidades de conservação do baixo Rio Negro e no Fórum de Apoio às Comunidades Rurais e Ribeirinhas de Manaus (FOPEC). E-mail: pebafopec@hotmail.com

Marcelo Moreira - Ex-técnico de Geoprocessamento da Fundação Vitória Amazônica. E-mail: pinguela@fva.org.br

Yara Camargo - Ex-técnica em Gestão de Áreas Protegidas da Secretaria de Desenvolvimento Sustentável do Estado do Amazonas (SDS) onde auxiliou no processo de implementação de unidades de conservação estaduais. E-mail: yaroca@gmail.com

\section{Resumo}

A revisão de limites e categorias de unidades de conservação têm se tornado comum na Amazônia brasileira. Entretanto, poucos estudos caracterizaram os eventos históricos e os papéis desempenhados por distintos atores regionais nos processos técnicos e políticos associados a estes eventos. Neste estudo foram investigados os eventos históricos subjacentes à reconfiguração de limites e categorias de dois Parques Estaduais no Estado do Amazonas. Foram registrados 56 eventos históricos e identificados 26 atores institucionais relevantes na gestão das duas unidades de conservação. A revisão dos limites e categoria se concretizou no Parque Estadual Rio Negro - Setor Sul. Por outro lado, as demandas sociais pouco estruturadas e um contexto desfavorável na política ambiental do Amazonas impediram a conclusão do processo no Parque Estadual do Rio Negro - Setor Norte. A revisão de limites e categorias pode ser uma estratégia válida de resolução parcial dos conflitos fundiários em unidades de conservação. Propostas desta natureza, no entanto, não devem ser banalizadas, mas baseadas em detalhadas análises técnico-científicas negociadas entre as comunidades locais, gestores e representantes políticos a fim de se evitar prejuízos na proteção da biodiversidade.

\section{Palavras-chave}

Áreas Protegidas. Amazônia. Regularização Fundiária. Compatibilidade de Gestão. Políticas Públicas.

\begin{abstract}
The revision of boundaries and categories of protected areas had been frequent in the Brazilian Amazon. However, few studies characterizing the historical events and the role of regional stakeholders associate to technical and political processes of revision of boundaries and categories of conservation units. In this study were investigate the historical events associated to change of limits and categories of two state parks in the Amazonas State. We recorded 56 historical events and identified 26 institutional stakeholders relevant to management of both protected areas. The process of revision of limits and category was finished in the Rio Negro State Park - Southern Sector. In contrast, unstructured social demands and an unfavorable context in the Amazonas State environmental policy impeding the conclusion of the processes in the Rio Negro State Park - Northern Sector. Change of the limits and categories of conservation units could be a valid strategy to partially solving the land conflicts in protected areas. Such proposals, however, needs be based in detailed technical and scientific analysis negotiated between local communities, managers, and political representation to avoid prejudices in the protection of biodiversity.
\end{abstract}

\section{Keywords}

Protected Areas. Amazon. Land Regulation. Managing Compatibility. Public Policies. 


\section{INTRODUÇÃO}

As unidades de conservação (UC) se constituem nas categorias de áreas protegidas mais complexas do Brasil em tamanho e diversidade nas formas de gestão. Atualmente, existem na Amazônia Legal 338 UC cobrindo pouco mais de 129 milhões de hectares (26\% do território da região), sendo 219 delas pertencentes ao grupo de uso sustentável e 119 de proteção integral. Os governos estadual e federal são responsáveis pela gestão de 193 e 145 UC, respectivamente (UNIDADES DE CONSERVAÇÃO NO BRASIL, s. d.).

Um sistema de áreas protegidas de tal magnitude e complexidade não poderia se estabelecer sem que conflitos de interesse fossem documentados na geografia regional. Devido à sua forma de gestão territorial, áreas oficialmente protegidas podem se tornar empecilhos parciais ou totais a projetos de desenvolvimento, a maioria planejada de forma exógena à Região Amazônica (BECKER, 2005; LITTLE, 2013). O aspecto mais concreto destes conflitos é a reconfiguração espacial e redefinição nas formas de gestão de UC na Amazônia brasileira.

Em anos recentes foram registrados vários atos dos poderes públicos nas esferas federal e estadual que implicaram na revisão de limites e categorias de UC, incluindo sua completa extinção (BERNARD; PENNA; ARAÚJO, 2014; MARQUES; PERES, 2015). Estes eventos se tornaram tão frequentes na Amazônia e em outras partes do mundo que receberam a denominação de PADDD (Protected Area Downgrading, Downsizing, and Degazettement) na literatura internacional (MASCIA; PAILLER, 2011). A expansão de eventos de revisão de limites e categorias de áreas protegidas tem alertado os conservacionistas para a fragilidade institucional destas áreas que se configuram como as principais estratégias de conservação da biodiversidade e ecossistemas em escala mundial (JUFFE-BIGNOLI et al., 2014).

Entre as principais causas dos eventos PAAD na Amazônia brasileira, estão: i) a ampliação de projetos para geração e distribuição de energia, especialmente a construção de usinas hidrelétricas; ii) demandas para o agronegócio; iii) assentamentos de comunidades rurais; e iv) conflitos de interesse com o setor privado (BERNARD; PENNA; ARAÚJO, 2014; MARQUES; PERES, 2015; PACK et al., 2016). Apesar das causas gerais subjacentes aos eventos de revisões de limites e categorias de UC na Amazônia brasileira terem sido identificadas, poucos estudos caracterizaram adequadamente os eventos históricos que, avaliando os papeis desempenhados, levam a estas revisões por distintos atores regionais nestes processos técnicos e políticos de negociação. Neste estudo, foram comparados os processos de reconfiguração de limites e categorias de 
duas unidades de conservação estaduais, localizadas na bacia do rio Negro, no estado do Amazonas: o Parque Estadual do Rio Negro - Setor Sul e o Parque Estadual do Rio Negro - Setor Norte.

Estas UC foram criadas antes da aprovação da Lei do Sistema Nacional de Unidades de Conservação (SNUC) (BRASIL, 2000) e ao longo do histórico de gestão dessas áreas, os conflitos entre as populações residentes e os órgãos ambientais se intensificaram, além de uma grande diversificação de atores que passaram a interagir com a gestão destas áreas protegidas (p. ex. indígenas, ribeirinhos, lideranças comunitárias, técnicos de órgãos públicos ambientais e entidades não governamentais).

Pretende-se com este estudo estabelecer uma melhor compreensão da dinâmica subjacente à configuração do extenso e complexo sistema de unidades de conservação existentes na Amazônia brasileira. Especificamente, o estudo foi desenhado para: i) comparar a trajetória histórica de gestão nas duas UC estaduais; ii) estabelecer uma narrativa temporal dos principais eventos que levaram à atual configuração espacial e de manejo das duas UC; e iii) identificar os atores institucionais e os seus papéis nos processos de revisão de limites e categorias destas áreas protegidas.

\section{MATERIAL E MÉTODOS}

\subsection{DEFINIÇÕES E ARCABOUÇO JURÍDICO}

As terminologias utilizadas neste estudo se baseiam em Mascia e Pailler (2011), para as definições originais, e Penna (2013), para suas respectivas correspondências na língua portuguesa. Estes autores definem os eventos associados à revisão de limites e categorias de áreas protegidas do seguinte modo:

- criação: ato de se criar, em qualquer esfera do poder público, uma unidade de conservação com limites e destinação previstos na Lei do SNUC ou leis análogas estaduais e municipais (MASCIA; PAILLER, 2011);

- declassificação: evento onde uma UC perde completamente seu status legal de área protegida. $\mathrm{Na}$ língua inglesa, este evento é denominado degazettement sendo entendido como a perda da proteção legal de toda a área protegida (MASCIA; PAILLER, 2011);

- redelimitação: revisão dos limites geográficos de uma UC como definidos no decreto de criação da mesma e que pode implicar em aumento, diminuição ou 
manutenção de área com mudança de forma. Redelimitação com diminuição da área protegida é conhecida como downsiz̨ing (MASCIA; PAILLER, 20011);

- reclassificação ou recategorização: evento em que uma UC sofre alteração de categoria ou de tipo, o que pode implicar na diminuição ou no aumento de restrição legal para atividades humanas. A diminuição de restrições legais em processos de reclassificação é denominada downgrading (MASCIA; PAILLER, 20011).

O arcabouço jurídico que dá sustentação aos atos do poder público acima definidos é providenciado pela Lei do Sistema Nacional de Unidades de Conservação (BRASIL, 2000). Especificamente em três parágrafos do Artigo 22, no Capítulo IV, o SNUC traz as seguintes orientações a respeito de revisão de limites e categorias de UC no Brasil:

[...] $\int 5^{\circ}$ As unidades de conservação do grupo de Uso Sustentável podem ser transformadas total ou parcialmente em unidades do grupo de Proteção Integral, por instrumento normativo do mesmo nível hierárquico do que criou a unidade, desde que obedecidos os procedimentos de consulta estabelecidos no $\int 2^{\circ}$ deste artigo;

$\int 6^{\circ}$ A ampliação dos limites de uma unidade de conservação, sem modificação dos seus limites originais, exceto pelo acréscimo proposto, pode ser feita por instrumento normativo do mesmo nível hierárquico do que criou a unidade, desde que obedecidos os procedimentos de consulta estabelecidos no $\int 2^{\circ}$ deste artigo;

$\int 7$ o A desafetação ou redução dos limites de uma unidade de conservação só pode ser feita mediante lei específica [...] (BRASIL, 2000, sem paginação).

No âmbito do estado do Amazonas, a Lei do Sistema Estadual de Unidades de Conservação (AMAZONAS, 2007) também trata da mesma matéria, sendo seus itens III e IV praticamente idênticos aos parágrafos quinto e sexto do SNUC mencionados acima. No entanto, no inciso V do artigo 28, o SEUC estabelece que:

V - A desafetação ou redução dos limites de uma unidade de conservação, bem como transformação de uma Unidade do grupo de Proteção Integral em Uso Sustentável dependem de lei específica e consulta pública (AMAZONAS, 2007, p. 8, grifo dos autores).

Além de prever os processos de revisão de limites, as leis do SNUC e do SEUC tratam, obviamente, dos processos de criação de UC e estabelecem que os mesmos devam ser precedidos de diagnósticos de campo e consultas públicas para a definição da categoria geral (proteção integral ou uso sustentável), específica (p. ex. Reserva Extrativista ou Parque Nacional) e de seus limites geográficos. As 
características socioambientais devem ser levadas em conta no ato de criação da UC, evitando assim a incompatibilidade de gestão com a realidade local. No entanto, em muitos casos, como os das UC analisadas neste estudo, a definição de limites e categorias não foi precedida de tais estudos técnicos e nem de consultas públicas aos moradores da região.

\section{2 ÁREA DE ESTUDO}

O estudo foi realizado na região do baixo curso do rio Negro, onde se localiza o Mosaico do Baixo Rio Negro (MBRN) reconhecido oficialmente pela Portaria $\mathrm{n}^{\circ}$ 483, de 14 de dezembro de 2010 (Figura 1). O MBRN ocupa uma área de mais de sete milhões de hectares divididos entre UCs estaduais, federais e municipais (Figura 1, Tabela 1). Além de estarem localizados dentro dos limites do MBRN, os parques estaduais analisados, também fazem parte da Reserva da Biosfera da Amazônia Central (Figura 1).

Figura 1 - Localização dos parques estaduais do rio Negro (Setor Note e Setor Sul) dentro dos limites da Reserva da Biosfera da Amazônia Central e do Mosaico do Baixo Rio Negro. A numeração das unidades de conservação do Mosaico do Baixo Rio Negro segue aquela da Tabela 1.

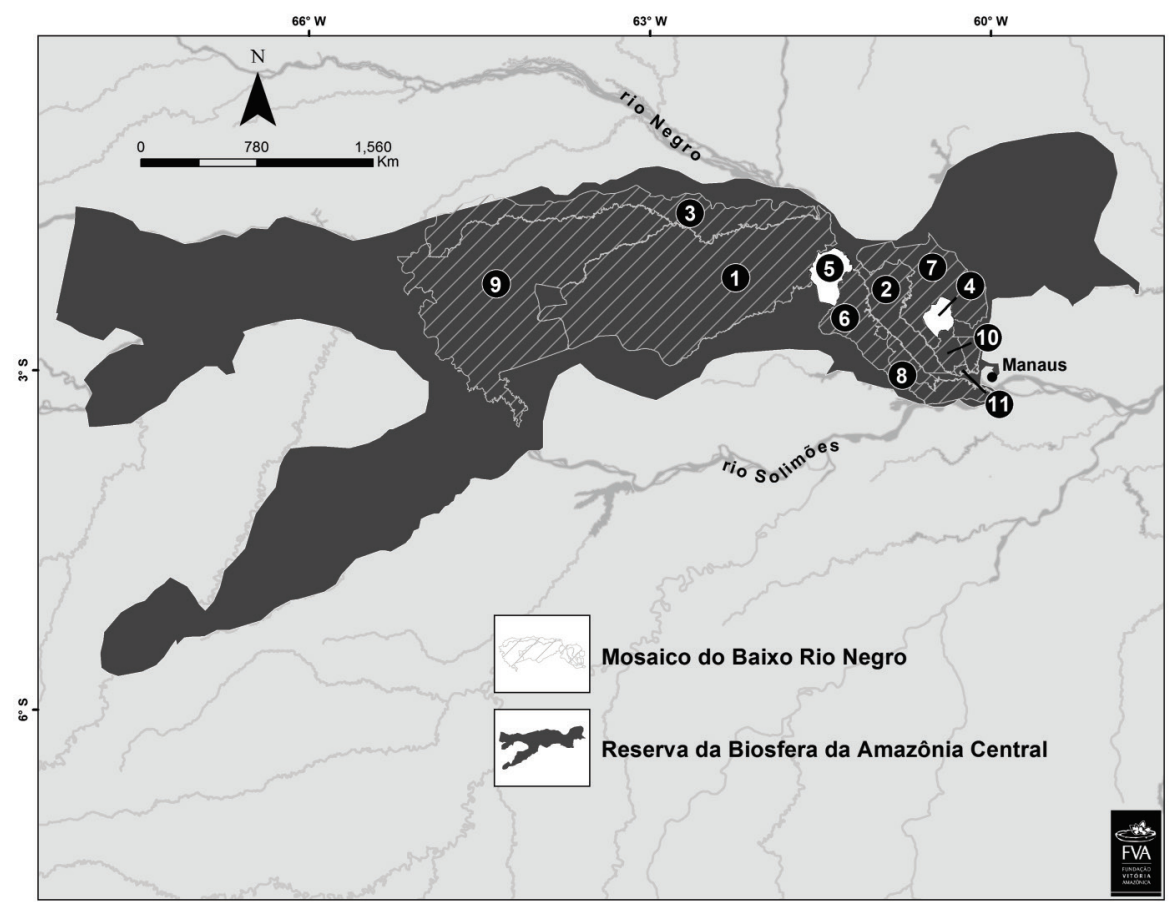

Fonte: FVA (s. d.). 
Tabela 1 - Unidades de conservação que fazem parte do Mosaico do Baixo Rio Negro (MBRN), Amazonas, Brasil.

\begin{tabular}{|c|c|c|}
\hline Unidades de conservação & $\begin{array}{l}\text { Ano de } \\
\text { criação }\end{array}$ & $\begin{array}{l}\text { Tamanho } \\
\text { (ha) }\end{array}$ \\
\hline \multicolumn{3}{|l|}{ Federais } \\
\hline 1. Parque Nacional do Jaú - PI* & 1980 & 2272,000 \\
\hline 2. Parque Nacional de Anavilhanas ${ }^{1}$ - PI & 2008 & 350018 \\
\hline 3. Reserva Extrativista do Rio Unini - US* & 2006 & 833352 \\
\hline Sub-total federais & & 3455,370 \\
\hline \multicolumn{3}{|l|}{ Estaduais } \\
\hline 4. Parque Estadual do Rio Negro - Setor Sul ${ }^{2}$ - PI & 1995 & 86601 \\
\hline 5. Parque Estadual do Rio Negro - Setor Norte - PI & 1995 & 146028 \\
\hline $\begin{array}{l}\text { 6. Área de Proteção Ambiental da Margem Direita do Rio Negro } \\
\text { - US }\end{array}$ & 1995 & 461741 \\
\hline $\begin{array}{l}\text { 7. Área de Proteção Ambiental da Margem Esquerda do Rio } \\
\text { Negro - US }\end{array}$ & 1995 & 611008 \\
\hline 8. Reserva de Desenvolvimento Sustentável do Rio Negro - US & 2008 & 103086 \\
\hline 9. Reserva de Desenvolvimento Sustentável do Amanã - US & 1998 & 2350,000 \\
\hline $\begin{array}{l}\text { 10. Reserva de Desenvolvimento Sustentável Puranga Conquista }{ }^{3} \\
\text { - US }\end{array}$ & 2014 & 76936 \\
\hline Sub-total estaduais & & 3835,400 \\
\hline \multicolumn{3}{|l|}{ Municipais } \\
\hline 11. Reserva de Desenvolvimento Sustentável do Tupé - US & 2005 & 11973 \\
\hline Sub-total municipais & & 11973 \\
\hline Total geral & & 7302,743 \\
\hline
\end{tabular}

Fonte: Unidades de Conservação do Brasil (s. d.).

Elaboração dos autores.

* PI = unidades de conservação de proteção integral, US = unidades de conservação de uso sustentável.

1) O Parque Nacional de Anavilhanas resultou de uma reclassificação da Estação Ecológica de Anavilhanas criada em 1981 cujos limites não foram alterados no processo de reclassificação. 2) $O$ tamanho da unidade segue o definido na Lei $\mathrm{n}^{\mathrm{O}} 4015$, de 24.03.2014. 3) A RDS Puranga Conquista formalmente não faz parte do MBRN como definido pela Portaria no 483, de 14 de dezembro de 2010. No entanto, os gestores e os representantes comunitários da reserva têm assento no Conselho do MBRN onde já está se discutindo a inserção formal desta unidade junto ao referido mosaico.

O Parque Estadual do Rio Negro - Setor Sul (doravante Parest Setor Sul) se localiza a cerca de $50 \mathrm{~km}$ de Manaus e, em seus limites redefinidos pelo Decreto nํㅡㄹ 2646, de 22.05.2001 ${ }^{1}$, ocupava uma área de cerca de 155.881 hectares, englobando quase a totalidade da bacia do rio Cuieiras (Figura 2). O Parest Setor

\footnotetext{
1 Os dois parques estaduais do rio Negro tiveram seus limites originais redefinidos em 2001 (ver resultados).
} 
Sul é vizinho do atual Parque Nacional de Anavilhanas ${ }^{2}$ e da Área de Proteção Ambiental Margem Esquerda do Rio Negro (Figura 2). Segundo o plano de gestão da UC, em 2010 existiam sete comunidades e cerca de 170 famílias vivendo no interior do Parest Setor Sul (AMAZONAS, 2010).

Figura 2 - Limites atuais do Parque Estadual do Rio Negro - Setor Sul (4), da Reserva de Desenvolvimento Sustentável Puranga Conquista (10) e do Parque Estadual do Rio Negro - Setor Norte (5).

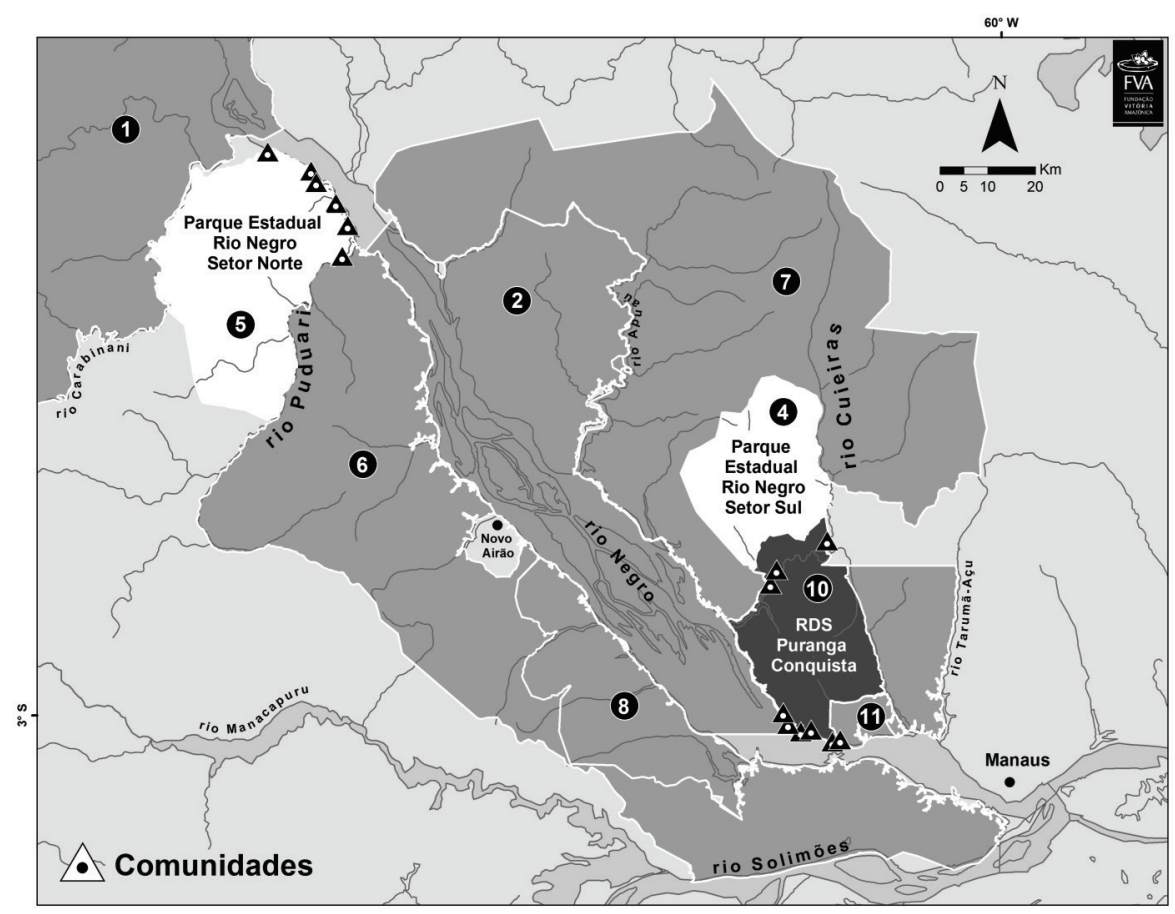

Fonte: FVA (s. d.).

Localizado mais ao norte e tendo parte de seus limites vizinhos ao Parque Nacional do Jaú, encontra-se o Parque Estadual do Rio Negro - Setor Norte (doravante Parest Setor Norte). O Parest Setor Norte possui atualmente 220.012 hectares e engloba uma parte significativa da bacia do rio Puduari (Figura 2). Comparado ao Parest Setor Sul, o número de famílias e comunidades do Parest

2 O Parque Nacional de Anavilhanas se tratava, originalmente, de uma estação ecológica até ser reclassificada em parque nacional pela Lei $\mathrm{n}^{\mathrm{o}}$ 11.799, de 29.10.2008. Neste evento de reclassificação não houve nenhuma modificação dos limites originais. Segundo a definição de Mascia e Pailler (2011), trata-se de um evento de diminuição de restrição legal ou downgrading, já que as estações ecológicas são mais restritivas às atividades humanas, não permitindo, por exemplo, atividades de uso público como turismo ecológico (BRASIL, 2000). 
Setor Norte é mais modesto, tendo sido identificadas, até 2006, 54 famílias distribuídas em seis comunidades no interior e entorno imediato da unidade (AMAZONAS, 2008).

\section{METODOLOGIA}

Este estudo foi baseado na reconstituição dos eventos que levaram à recategorização e redelimitação dos parques estaduais do rio Negro e em uma análise desta trajetória a partir da visão de distintos atores. As ações e discussões de gestão sobre as UC em análise se basearam em complexas inter-relações técnicas e políticas envolvendo inúmeros atores institucionais. Um dos desafios do estudo foi estabelecer uma narrativa lógica desses eventos que qualificasse os distintos atores institucionais e seus papéis nos processos de revisão dos limites e categorias das UC.

A reconstituição histórica foi subsidiada por uma cuidadosa e abrangente revisão de documentos oficiais. Nesta análise documental, buscou-se por informações e fatos relevantes para a gestão das UC em análise incluindo datas, eventos e entidades associadas a estes eventos. Os documentos mais relevantes consultados para o estudo foram: i) os decretos de criação e revisão de limites das UC; ii) os planos de gestão das UC; iii) as atas de reunião dos Conselhos Gestores; iv) as atas de reunião do Conselho do Mosaico do Baixo Rio Negro $(\mathrm{MBRN}), \mathrm{v})$ as atas de reunião do Conselho da Reserva da Biosfera da Amazônia Central (CERBAC); e vi) os processos jurídicos de encaminhamento da revisão de limites e categorias.

Estes documentos são estratégicos e incluem os encaminhamentos formais mais relevantes no histórico de gestão das UC em análise. No entanto, é provável que eventos não documentados tenham relevância para o histórico de gestão dos parques, sendo necessária uma estratégia de identificação de tais eventos informais. Para esta busca de eventos informais relevantes também foram consultados relatórios técnicos, sites da rede mundial de computadores e entrevistas não estruturadas com atores relevantes para a gestão das UC, buscando tornar mais completo possível o relato de determinado conjunto de fatos.

As instituições identificadas durante os levantamentos documentais e informais de eventos que influenciaram direta ou indiretamente a gestão das 
duas UC foram avaliadas quanto ao seu papel nestes processos. Foi realizada uma pesquisa de opinião entre técnicos de instituições que participaram ativamente da gestão das duas UC. Aos participantes desta enquete foi solicitado que avaliassem a relevância de instituições previamente identificadas e lhes aplicasse as seguintes notas:

- Nota 0 (N0): instituição que, apesar de ter sido importante para o histórico de gestão das UC, não teve nenhuma contribuição direta ou indireta no processo de revisão de limite ou categoria da UC em questão;

- Nota 1 (N1): instituição que contribuiu de modo pontual e indireto no processo de revisão de limite ou categoria da UC em questão;

- Nota 2 (N2): instituição que contribuiu de modo mais direto, técnica ou politicamente, no processo de revisão de limite ou categoria da UC em questão, mas cuja contribuição se deu de forma pontual;

- Nota 3 (N3): instituição que acompanhou todo o histórico e ofereceu efetivas contribuições técnicas ou políticas ao longo do processo de revisão de limite ou categoria da UC em questão;

- Não avaliada (NA) - situação em que o colaborador julga não possuir elementos necessários para uma avaliação adequada da contribuição de determinada instituição para o processo de revisão de limite ou categoria da UC em questão.

Para sintetizar os resultados desta avaliação, os atores institucionais foram categorizados em: i) órgãos governamentais; ii) academia; iii) coletivos de gestão; iv) financiadores; v) organizações de base comunitária; vi) classe política; e vii) entidades do terceiro setor. As notas dadas pelos avaliadores foram transformadas em proporções para comparar a contribuição relativa de cada categoria de ator.

Para esta avaliação foram consultados técnicos de órgãos governamentais $(n=3)$, entidades do terceiro setor $(n=3)$ e lideranças comunitárias $(n=1)$ que participaram ativamente da gestão das UC estudadas. Enfatizamos que o baixo número de pessoas envolvidas nesta avaliação foi parcialmente compensado pela extensa experiência e envolvimento das mesmas nas articulações técnicas e políticas relacionadas à revisão dos limites e das categorias dos dois parques. 


\section{RESULTADOS}

\subsection{BREVE CONTEXTUALIZAÇÃO DO HISTÓRICO DE GESTÃO DOS PARQUES ESTADUAIS DO RIO NEGRO}

Os parques estaduais do rio Negro foram criados em 1995 por ato do então governador do estado do Amazonas Amazonino Mendes, em cerimônia que contou com a presença do ex-presidente da República Fernando Henrique Cardoso e da ex-ministra de Indústria e Comércio Doroteia Werneck. Os instrumentos de criação das UC foram os decretos no 16.497 e 16.498, de 02 de abril de 1995, que formalizaram o seguinte conjunto de UC: o Parest Setor Norte (178.620 ha), o Parest Setor Sul (257.422 ha), a Área de Proteção Ambiental (APA) da Margem Direita do Rio Negro e a Área de Proteção Ambiental da Margem Esquerda do Rio Negro (Figura 3A).

Segundo os decretos originais, os parques estaduais do rio Negro foram criados tendo como objetivo "a preservação de ecossistemas naturais de grande relevância ecológica e beleza cênica, possibilitando a realização de pesquisas científicas e o desenvolvimento de atividades de educação e interpretação ambiental, de recreação em contato com a natureza e de turismo ecológico" (UNIDADES DE CONSERVAÇÃO NO BRASIL, s. d., s. p.).

Em 2001, o mesmo governador Amazonino Mendes assina o Decreto no 2.646 que altera os limites dos parques e das áreas de proteção ambiental (Figura 3B). O Parest Setor Norte teve sua área diminuída para 146.028 ha e o Parest Setor Sul para 157.807 ha. Além de terem suas áreas diminuídas, os dois parques passaram a se localizar em margens opostas do Rio Negro (Figura 3B). As áreas de proteção ambiental ficaram com 586.422 ha (APA da Margem Esquerda) e 566.365 ha (APA da Margem Direita). As principais motivações para estes ajustes de área e localização geográfica foram as sobreposições entre os limites originais dos parques com outras áreas protegidas vizinhas como o Parque Nacional do Jaú, a Estação Ecológica de Anavilhanas e a Terra Indígena Waimiri-Atroari. 
Figura 3 - Evolução histórica dos limites e categorias das unidades de conservação estaduais do baixo Rio Negro. A) Limites dos parques estaduais e áreas de proteção ambiental segundo o Decreto $\mathrm{n}^{\circ}$ 16.497, de 02 de abril de 1995; B) Limites dos parques estaduais e das áreas de proteção ambiental revistos segundo o Decreto no 2646, de 22 de maio de 2001; C) Revisão de limites do Parque Estadual Setor Sul e criação da Reserva de Desenvolvimento Sustentável Puranga Conquista segundo o Decreto nº 4015, de 24 de março de 2014; D) Proposta de revisão de limites do Parque Estadual do Rio Negro Setor Norte e criação de uma nova unidade de conservação aprovada em consulta pública realizada em dezembro de 2014.
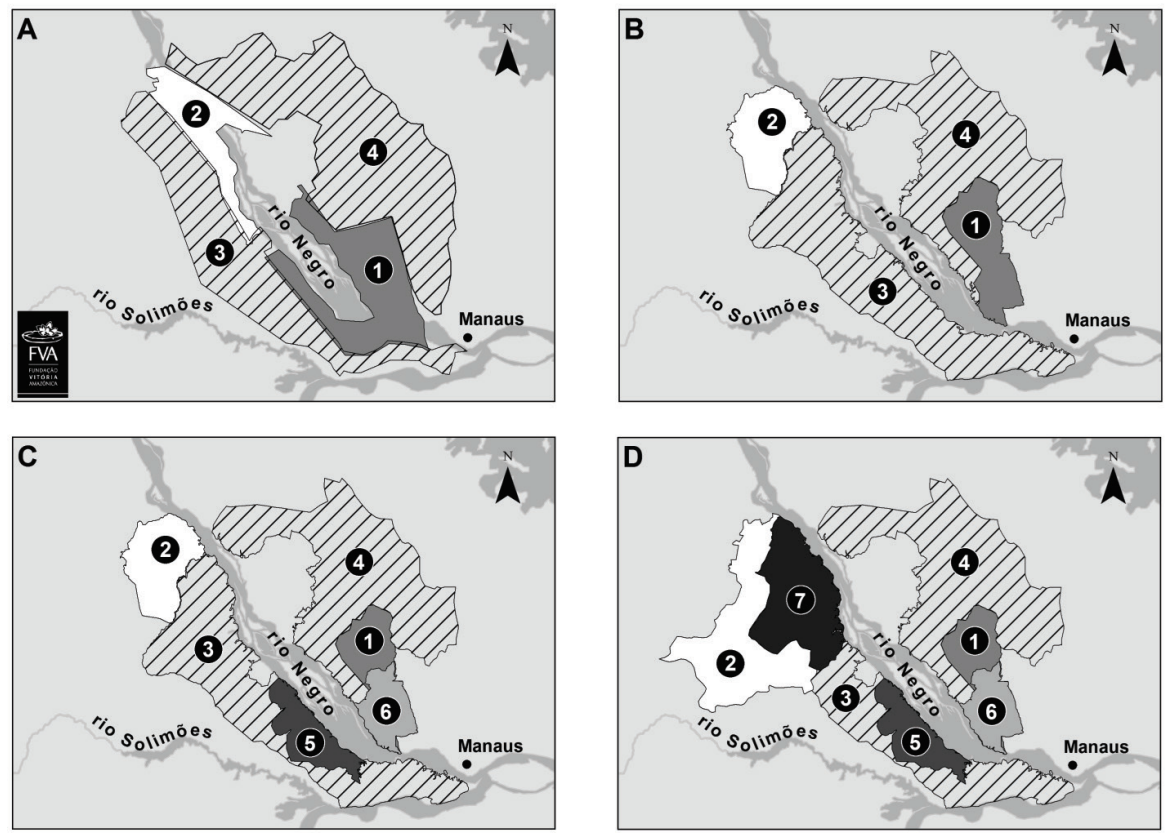

Fonte: FVA (s. d.)

Legendas para os nomes das unidades de conservação: 1) Parque Estadual do Rio Negro - Setor Sul; 2) Parque Estadual do Rio Negro - Setor Norte (que deveria ser rebatizado de Parest Velho Airão); 3) Área de Proteção Ambiental Margem Direita do Rio Negro; 4) Área de Proteção Ambiental Margem Esquerda do Rio Negro; 5) Reserva de Desenvolvimento Sustentável do Rio Negro; 6) Reserva de Desenvolvimento Puranga Conquista; 7) Reserva de Desenvolvimento Sustentável Bom Jesus do Puduari.

Em meados dos anos 2000, deu-se início aos trabalhos para elaboração dos planos de gestão dos parques estaduais do rio Negro. Estes planos de gestão foram elaborados pela Secretaria Estadual de Meio Ambiente e Desenvolvimento Sustentável (SDS), em parceria com organizações ambientalistas que já atuavam na região, especificamente a Fundação Vitória Amazônica (FVA) no Parest Setor Norte e o Instituto de Pesquisas Ecológicas (IPÊ) no Parest Setor Sul. 
Os primeiros trabalhos de campo destas entidades revelaram a existência de comunidades com inúmeras famílias residindo dentro dos limites dos parques, configurando um claro conflito com a gestão, uma vez que não é permitida a presença humana em unidades de conservação de proteção integral, segundo as leis federal (SNUC) e estadual (SEUC).

O mais recente evento na história dos parques estaduais do rio Negro foi a redelimitação do Parest Setor Sul (que passou a contar com 77.950,86 ha) e a reclassificação de parte de seu território na Reserva de Desenvolvimento Sustentável (RDS) Puranga Conquista (86.235 ha), criada pela Lei no 4.015, de 24.03.2014 (Figura 3C). Também, em dezembro de 2014, foi realizada uma consulta pública com a participação de lideranças, gestores públicos, parceiros de gestão e comunitários que deliberaram pela revisão de limites do Parest Setor Norte e criação de outra reserva de desenvolvimento sustentável (Figura 3D). Os encaminhamentos e deliberações desta consulta pública, no entanto, não resultaram na revisão formal dos limites do Parest Setor Norte, nem na criação de uma nova UC, conforme podemos observar na discussão realizada na próxima seção.

\subsection{HISTORIOGRAFIA DA GESTÃO DOS DOIS PARQUES ESTADUAIS DO BAIXO RIO NEGRO}

Durante os levantamentos documentais, foram identificados 56 eventos relevantes para a evolução no processo de gestão das duas UC estudadas ${ }^{3}$. Estes eventos foram classificados como de contextos mais amplos ou específicos e que afetaram diretamente determinada UC ou ambas. Estes eventos foram organizados em períodos de cinco anos e nota-se um avanço gradual nas dinâmicas de gestão das duas UC ao longo dos anos. Uma análise mais detalhada do conjunto de eventos históricos que permite identificar períodos distintos de evolução na gestão das duas UC é descrita abaixo.

Período 1 (1995-1999) - Origem dos parques e conflitos: este período se refere ao processo de consolidação de uma política ambiental ainda bastante inicial por parte do Governo do Amazonas. Esta política ambiental se torna mais visível com a criação do Instituto de Proteção Ambiental do Amazonas (IPAAM) e a criação de um conjunto de unidades de conservação no baixo curso do rio Negro (Figura 3A). Ao final deste período, foram registrados eventos de conflitos entre os moradores do rio Cuieiras (residentes no interior do Parest Setor Sul) e fiscais do Instituto Brasileiro do Meio Ambiente e dos Recursos

3 A lista completa e mais detalhada acerca destes eventos pode ser obtida diretamente com os autores. 
Naturais Renováveis (IBAMA) que atuavam na gestão da vizinha Estação Ecológica de Anavilhanas (Figura 2). Também, nesta mesma época algumas comunidades indígenas que se instalaram no rio Cuieiras se organizaram para solicitar o reconhecimento de seus territórios como terras indígenas junto à Fundação Nacional do Índio (FUNAI).

Período 2 (2000-2004) - Início da gestão dos parques: este período foi marcado por grandes mudanças contextuais tanto na esfera federal (p. ex. aprovação do SNUC) quanto estadual (p. ex. criação da SDS). Na escala mais local, ainda se registraram eventuais conflitos entre comunitários do Parest Setor Sul e o IPAAM e IBAMA. Estes conflitos tenderam a diminuir com a inserção de novos atores na gestão dos parques. Entidades parceiras do IPAAM e posteriormente da SDS, entram no cenário com objetivo de elaborar os planos de gestão dos parques. A Fundação Vitória Amazônica (FVA) inicia os estudos técnicos que iriam subsidiar o plano de gestão do Parest Setor Norte e o Instituto de Pesquisas Ecológicas (IPÊ) desenvolve atividades similares no contexto do Parest Setor Sul. Este período também foi marcado pelo lançamento do Programa Áreas Protegidas da Amazônia (ARPA), que teve e continua tendo enorme relevância na consolidação do sistema de unidades de conservação da Amazônia. Já em 2004, os dois parques estaduais do rio Negro passam a contar com recursos financeiros do ARPA para a sua gestão, apoio este que perdura até os dias atuais.

\section{Período 3 (2005-2009) - Amadurecimento da gestão dos parques:} período de grandes avanços na política estadual de meio ambiente, onde o estado do Amazonas passa a contar com uma legislação específica associada às unidades de conservação, o Sistema Estadual de Unidades de Conservação (SEUC). Na mesma lei é prevista a criação de um Centro Estadual de Unidades de Conservação (CEUC). Avanços também foram observados na destinação de recursos financeiros para a gestão das UC do Amazonas. Além dos recursos negociados em longo prazo com o Programa ARPA, a SDS passa a contar com apoio financeiro da Fundação Gordon \& Betty Moore. O projeto Corredores Ecológicos oferece apoio financeiro a projetos de pequeno porte implementados nos dois parques estaduais pela FVA e pelo IPÊ. A instalação de um escritório da SDS no município de Novo Airão permitiu que fosse designado um conjunto de técnicos especificamente associados à gestão das duas unidades. Ao final deste período foi aprovado o plano de gestão do Parest Setor Norte (AMAZONAS, 2008) onde constava uma proposta de revisão de limites da unidade. Também foi realizada a primeira reunião do Conselho Gestor do Parest Setor Norte. Outros dois eventos que merecem 
destaque neste período foram: a criação do Projeto de Assentamento Sustentável (PDS) Cuieiras-Anavilhanas pelo INCRA, em 2005, dentro dos limites do Parest Setor Sul, e a realização de um seminário promovido pela FVA, em que se discutiu a redelimitação de UC e os termos de compromisso como estratégias para se avançar na regularização fundiária das UCs de proteção integral.

Período 4 (2010-2014) - Gestão territorial em larga escala: este foi o período onde aconteceram os eventos mais estratégicos nos processos de revisão dos limites e categorias das duas UC em análise. No contexto do Parest Setor Sul, foi aprovado o plano de gestão que também trazia uma proposta de revisão dos limites da UC (AMAZONAS, 2010). Logo após a aprovação do plano, foram realizadas intensas articulações entre os representantes comunitários, gestores formais e parceiros da UC e representantes da Assembleia Legislativa do Estado do Amazonas (ALEAM) no sentido de avaliar e aprovar a proposta de revisão dos limites do Parest Setor Sul.

Neste período, o Instituto Nacional de Colonização e Reforma Agrária (INCRA) se posicionou contra a redefinição dos limites da UC na primeira consulta pública (2011) por questões relacionadas à dominialidade da terra na região do parque. Este posicionamento foi revisto após negociações com a SDS e na segunda consulta pública (2012), o INCRA passou a apoiar a proposta e deu início ao processo de permuta de dominialidade de terra entre o ente federal (INCRA) e o estadual (SDS).

Estas articulações políticas institucionais se estenderam de 2010 até 2014 quando foi aprovada a Lei que modifica os limites e categorias da unidade de conservação (Figura 3C) pela ALEAM. É importante destacar que durante este período, os representantes das comunidades do Parest Setor Sul (Fórum de Apoio as Comunidades Rurais e Ribeirinhas de Manaus (FOPEC) e a Associação de Moradores do Parque), a chefia da UC e o principal parceiro na gestão da unidade (IPÊ) mantiveram uma contínua e consistente articulação com foco na negociação da revisão de limites da UC tanto junto ao órgão gestor (SDS) quanto aos representantes da classe política, especialmente a Comissão de Meio Ambiente da ALEAM.

No Parest Setor Norte, o processo avançou a partir de um evento que ocorreu em um contexto bastante diferenciado. Em 2012, o Governo do Amazonas anunciou a construção de uma cidade universitária da Universidade do Estado do Amazonas (UEA), no município de Iranduba, dentro dos limites da APA da Margem Direita do Rio Negro (Figura 2). A questão da revisão dos limites do Parest Setor Norte, cuja discussão não tinha avançado até então, foi 
levantada como uma opção de compensação ambiental aos impactos que a construção da cidade universitária iria causar.

Esta discussão se deu nas reuniões do Conselho da Biosfera da Amazônia Central (CERBAC) que designou um Grupo de Trabalho (GT) para avaliar as opções de compensação aos impactos ambientais da cidade universitária. Em 2014, o GT apresentou os resultados desta análise em reunião do Conselho do CERBAC onde se apontavam regiões propícias à criação de novas UC nas proximidades da futura cidade universitária e na região mais distante ao norte, onde se localiza o Parest Setor Norte, área em que se propunha a redelimitação da UC (Figura 2). A revisão dos limites do Parest Setor Norte e a criação de uma nova UC na região do rio Puduari foram sugeridas por este GT em uma proposta técnica similar à registrada no plano de gestão da unidade aprovada em 2008 (AMAZONAS, 2008). Os resultados deste estudo foram apresentados também no Conselho do Mosaico de Baixo Rio Negro (MBRN), que conta com representantes das comunidades do Parest Setor Norte.

Membros do Conselho do MBRN, representantes comunitários, gestores do Parest Setor Norte e técnicos de entidades parceiras (FVA e IPÊ) passaram, então, a discutir com os comunitários residentes dentro e no entorno do Parest Setor Norte, a proposta encaminhada pelo GT do CERBAC. Estas articulações com as comunidades locais culminaram com uma consulta pública realizada em dezembro de 2014, que contou com a presença da Secretária de Meio Ambiente do Amazonas, momento em que se debateu a proposta de revisão dos limites do Parest Setor Norte e a criação de uma nova UC na vizinhança imediata do parque (Reserva de Desenvolvimento Sustentável Bom Jesus do Puduari). Nesta consulta pública também se deliberou que o Parest Setor Norte deveria ter seu nome alterado para Parest Velho Airão (Figura 3D).

\section{Período 5 (2015-2016) - Mudanças de rumos na política ambiental}

do Amazonas: a reestruturação da Secretaria de Estado de Meio Ambiente e Desenvolvimento Sustentável do Amazonas (SDS), no início do ano de 2015, foi o evento que mais afetou os processos de gestão das UC estaduais em geral, e a revisão de limites e categoria do Parest Setor Norte especificamente. Em março de 2015, o governador em exercício do Amazonas determinou uma ampla reestruturação das Secretarias de Estado com foco em diminuição de gastos da máquina pública. Esta determinação atingiu a SDS que passou a ser denominada Secretaria de Estado do Meio Ambiente (SEMA). A antiga secretária da SDS que acompanhou de perto as negociações de revisão dos limites dos parques estaduais do rio Negro foi exonerada do cargo durante esta reestruturação. 
O efeito mais direto desta mudança na gestão das UC do Amazonas foi a extinção do Centro Estadual de Mudanças Climáticas (CECLIMA) e do Centro Estadual de Unidades de Conservação (CEUC). Estes dois centros foram fundidos no Departamento de Mudanças Climáticas e Gestão de Unidades de Conservação (DEMUC), o que implicou na diminuição do número de técnicos associados à gestão das UC estaduais. Esta diminuição de recursos humanos ligados diretamente à gestão das UC, no entanto, não atingiu as UC estaduais da calha do rio Negro. Os recursos negociados junto ao Programa Áreas Protegidas da Amazônia (ARPA) também continuaram garantindo a continuidade das atividades de gestão do Parest Setor Norte e Parest Setor Sul, este último com seus novos limites já definidos.

Todo este processo de mudanças na estrutura interna do órgão ambiental do estado se deu somente três meses após a consulta pública para revisão dos limites do Parest Setor Norte e criação de uma nova UC na região. Esta dinâmica contextual interna ao órgão acabou por protelar a finalização do processo negociado na consulta pública. No início de 2015, antes da reestruturação interna ter se concretizado, a Procuradoria Geral do Estado (PGE) havia solicitado da SDS, um memorial descritivo dos novos limites do Parest Setor Norte e da nova RDS a ser criada. A finalização deste documento não foi concretizada devido à intensa mudança no cargo de coordenação do DEMUC que deveria ter consolidado este processo.

Outro aspecto relevante foi a desmobilização do Conselho do CERBAC que foi de grande importância na concepção da proposta de compensação ambiental da cidade universitária da UEA (ver acima). O Conselho do CERBAC sempre foi bastante ativo no encaminhamento de decisões junto à SDS. Infelizmente, este conselho se desmobilizou após o encerramento do Projeto Corredores Ecológicos e não se reúne desde 2014.

Devido ao atraso na elaboração do Memorial Descritivo da UC, não foi possível acionar os agentes políticos (p. ex. Casa Civil, Comissão de Meio Ambiente) para a negociação da lei de revisão dos limites do Parest Setor Norte e criação da RDS Bom Jesus do Puduari junto à ALEAM.

\subsection{MAPEAMENTO DE ATORES}

Foram identificados 26 atores institucionais que afetaram direta ou indiretamente a gestão dos dois parques e que contribuíram em diversos níveis com o processo de revisão de limites e categoria dos mesmos (Tabela 2). Estes atores institucionais se distribuíram entre: órgãos governamentais ( $\mathrm{n}=10$ 
entidades); academia ( $\mathrm{n}=3)$; coletivos de gestão $(\mathrm{n}=3)$, financiadores $(\mathrm{n}=3)$; organizações de base comunitária $(n=3)$; classe política $(n=2)$ e entidade do terceiro setor $(\mathrm{n}=2)$.

Uma análise comparativa mostra as diferenças e as similaridades no papel de cada um destes atores, segundo a perspectiva dos avaliadores. Os grupos de atores que se destacaram de modo positivo (notas 2 ou 3) nos dois parques foram os coletivos de gestão, os financiadores e as entidades do terceiro setor (Figura 4).

Figura 4 - Resultado da avaliação dos grupos de atores institucionais que contribuíram com o processo de revisão de limites e categorias de dois parques estaduais do baixo rio Negro. A contribuição de cada grupo de atores está representada em proporção e as definições do sistema de notas são explicadas na metodologia. Os círculos interrompidos mostram as percentagens mais altas.

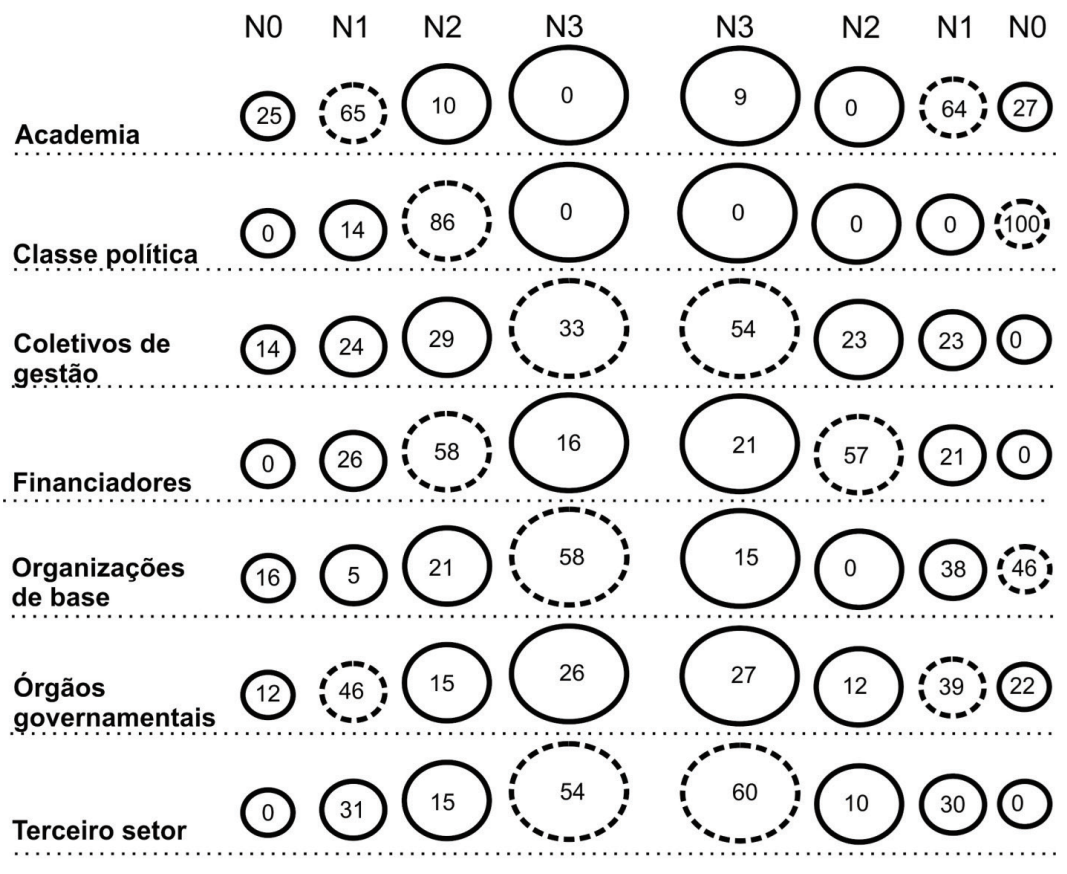

Parest Setor Sul

Parest Setor Norte

Elaboração dos autores, com base nos dados levantados na pesquisa.

Em contraste, alguns grupos de entidades tiveram pouca influência nos processos de revisão de limites e categorias das UC (notas 0 ou 1) como entidades acadêmicas e vários órgãos governamentais. Destaca-se, no entanto, que entre os órgãos públicos ambientais, destacaram-se entidades com avaliações mais positivas como a chefia das UC, conforme demonstra a Tabela 2. 
Tabela 2 - Notas dos avaliadores para os atores institucionais que afetaram o processo de revisão de limites e categorias dos dois parques estaduais do rio Negro.

\begin{tabular}{|c|c|c|c|c|c|c|c|c|c|c|}
\hline \multirow[t]{2}{*}{ Entidades } & \multicolumn{5}{|c|}{\begin{tabular}{|c|} 
Parest Rio Negro \\
- Setor Sul \\
(7 avaliadores) \\
\end{tabular}} & \multicolumn{5}{|c|}{$\begin{array}{c}\text { Parest Rio Negro } \\
\text { - Setor Norte } \\
\text { (5 avaliadores) }\end{array}$} \\
\hline & 0 & 1 & 2 & 3 & NA & 0 & 1 & 2 & 3 & NA \\
\hline Academia & 3 & 5 & 2 & 0 & & 2 & 3 & 0 & 1 & \\
\hline Universidade Federal do Amazonas (UFAM) & 2 & 5 & & & & 2 & 2 & & & 1 \\
\hline $\begin{array}{l}\text { Universidade do Estado do Amazonas } \\
\text { (UEA) }\end{array}$ & 3 & 3 & & & 1 & & 2 & & 1 & 2 \\
\hline $\begin{array}{l}\text { Instituto Nacional de Pesquisas da Amazônia } \\
\text { (INPA) }\end{array}$ & & 5 & 2 & & & 1 & 3 & & & 1 \\
\hline Classe política & 0 & 2 & 7 & 0 & & 3 & 0 & 0 & 0 & \\
\hline Comissão de Meio Ambiente & & & 7 & & & 3 & & & & 2 \\
\hline $\begin{array}{l}\text { Assembleia Legislativa do Estado do } \\
\text { Amazonas (ALEAM) }\end{array}$ & & 2 & 5 & & & 3 & & & & 2 \\
\hline Coletivos de gestão & 3 & 3 & 3 & 6 & & 0 & 2 & 2 & 5 & \\
\hline $\begin{array}{l}\text { Conselhos gestores das unidades de } \\
\text { conservação }\end{array}$ & & & 1 & 6 & & & 1 & 2 & & 2 \\
\hline $\begin{array}{l}\text { Conselho da Reserva da Biosfera da } \\
\text { Amazônia Central (CERBAC) }\end{array}$ & 3 & 2 & 2 & & & & & & 5 & \\
\hline $\begin{array}{l}\text { Conselho Mosaico do Baixo Rio Negro } \\
\text { (MBRN) }\end{array}$ & & 3 & 3 & 1 & & & 2 & 1 & 2 & \\
\hline Financiadores & 0 & 3 & 5 & 1 & & 0 & 1 & 3 & 1 & \\
\hline Projeto Corredores Ecológicos & & 3 & 2 & 1 & 1 & & 1 & 2 & 1 & 1 \\
\hline $\begin{array}{l}\text { Programa Áreas Protegidas da Amazônia } \\
\text { (ARPA) }\end{array}$ & & 1 & 4 & 1 & 1 & & 1 & 3 & 1 & \\
\hline Fundação Gordon \& Betty Moore (FBM) & & 1 & 5 & 1 & & & 1 & 3 & 1 & \\
\hline Organizações de base & 3 & 1 & 2 & 6 & & 2 & 2 & 0 & 1 & \\
\hline $\begin{array}{l}\text { Fórum de Apoio as Comunidades Rurais e } \\
\text { Ribeirinhas de Manaus (FOPEC) }\end{array}$ & & & 1 & 6 & & 2 & 2 & & 1 & \\
\hline $\begin{array}{l}\text { Comissão de Moradores do Parest - Setor } \\
\text { Sul }\end{array}$ & & & 2 & 5 & & 2 & 1 & & & 2 \\
\hline $\begin{array}{l}\text { Associação dos Moradores do Parest - Setor } \\
\text { Norte }\end{array}$ & 3 & 1 & 1 & & 2 & 2 & 2 & & 1 & \\
\hline Órgãos governamentais & 3 & 7 & 3 & 7 & & 2 & 4 & 2 & 3 & \\
\hline $\begin{array}{l}\text { Instituto de Proteção Ambiental do } \\
\text { Amazonas (IPAAM) }\end{array}$ & & 7 & & & & 1 & 4 & & & \\
\hline $\begin{array}{l}\text { Instituto Brasileiro do Meio Ambiente e dos } \\
\text { Recursos Naturais Renováveis (IBAMA) }\end{array}$ & 1 & 4 & & & 2 & 1 & 3 & & & 1 \\
\hline $\begin{array}{l}\text { Instituto Nacional da Colonização e Reforma } \\
\text { Agrária (INCRA) }\end{array}$ & & 1 & 3 & 3 & & 2 & & & & 3 \\
\hline Fundação Nacional do Índio (FUNAI) & 1 & 5 & 1 & & & 2 & 1 & & & 2 \\
\hline $\begin{array}{l}\text { Secretaria Estadual de Meio Ambiente e } \\
\text { Desenvolvimento Sustentável (SDS) }\end{array}$ & & & 3 & 4 & & & 1 & 1 & 3 & \\
\hline
\end{tabular}




\begin{tabular}{|c|c|c|c|c|c|c|c|c|c|c|}
\hline Marinha do Brasil & 3 & 3 & & & 1 & & 3 & 2 & & \\
\hline $\begin{array}{l}\text { Instituto de Desenvolvimento Agropecuário } \\
\text { e Florestal do Amazonas (IDAM) }\end{array}$ & 1 & 6 & & & & 2 & 1 & & & 2 \\
\hline $\begin{array}{l}\text { Centro Estadual de Unidades de } \\
\text { Conservação (CEUC) }\end{array}$ & & 1 & 3 & 3 & & & 1 & 1 & 3 & \\
\hline Chefia das UC & & & & 7 & & & 1 & 1 & 3 & \\
\hline $\begin{array}{l}\text { Secretaria de Estado do Meio Ambiente } \\
\text { (SEMA) }\end{array}$ & 2 & 3 & & & 2 & 1 & 1 & & 2 & 1 \\
\hline \multicolumn{11}{|l|}{ Terceiro setor } \\
\hline Fundação Vitória Amazônica (FVA) & & 4 & 1 & 1 & 1 & & & & 5 & \\
\hline Instituto de Pesquisas Ecológicas (IPÊ) & & & 1 & 6 & & & 3 & 1 & 1 & \\
\hline
\end{tabular}

Elaboração dos autores, com base nos dados levantados na pesquisa.

Os maiores contrastes entre as duas UC se verificaram na avaliação do papel das entidades de base comunitária e da classe política (Figura 4). Entidades de base comunitária foram muito bem avaliadas no Parest Setor Sul, mas não no Parest Setor Norte, conforme também pode ser constatado na Figura 4. A classe política foi mal avaliada no caso do Parest Setor Norte, muito provavelmente porque membros deste grupo de atores não foram devidamente acionados para a finalização do processo de revisão de limites e categoria desta UC.

\section{DISCUSSÃO}

\subsection{CONTEXTOS DIFERENCIADOS PARA REVISÃO DE LIMITES E CATEGORIAS DE UC}

Os casos de revisão de limites e categorias de UC na Amazônia brasileira, em sua maioria, foram motivados por demandas políticas com destaque para projetos de geração e distribuição de energia e agronegócios (BERNARD; PENNA: ARAÚJO, 2014; PACK et al., 2016). Os casos analisados neste estudo, em contraste, nasceram de uma demanda social negociada em função da situação de irregularidade da presença humana em unidades de conservação de proteção integral.

Como já destacado, a criação dos parques estaduais do rio Negro não foi precedida de consulta pública ou subsidiada por estudos técnico-científicos que apontassem as melhores opções de categorias e/ou limites de áreas protegidas para a região. Os conflitos entre a gestão pública e as comunidades locais surgiram como problemática mais concreta a partir da construção coletiva dos planos de gestão das UC. Desde o início dos trabalhos de campo nas UC, em meados dos 
anos 2000, os parceiros de gestão (FVA e IPÊ) e representantes do órgão gestor (SDS) identificaram as necessidades de revisão de limites e categorias nestas áreas. No entanto, foi somente com o amadurecimento da prática de gestão que estas estratégias foram efetivamente colocadas em prática.

Ressalta-se que mesmo compartilhando alguns eventos contextuais semelhantes, os dois parques apresentaram diferenças no resultado final das negociações. No início de 2014, o Parest Setor Sul foi formalmente redelimitado e parte de seu território transformado na RDS Puranga Conquista. Os atores envolvidos conseguiram cumprir e negociar todas as etapas necessárias previstas em lei para a conclusão do processo. O mesmo não aconteceu no Parest Setor Norte, apesar das negociações terem avançado até a realização de uma consulta pública. Nesse sentido, que condicionantes históricos podem explicar estas trajetórias diferenciadas no resultado final?

Um conjunto bastante diverso de atores institucionais participou do processo de gestão das duas UC em análise. Entretanto, a contribuição relativa destes atores foi bastante variada dentro e entre as UC. O Parest Setor Sul contou desde o início com uma sólida interação entre uma entidade parceira de gestão (IPE) , a chefia da unidade e as lideranças comunitárias locais. Este trio de atores permaneceu focado na revisão dos limites e categoria do Parest Setor Sul, por entender que esta seria a ação prioritária de maior alcance prevista no plano de gestão da unidade. Deste modo, conseguiram negociar o encaminhamento desta demanda junto ao órgão gestor ambiental (SDS) e a classe política local, representada principalmente pela Comissão de Meio Ambiente da Assembleia Legislativa do Estado do Amazonas.

Além da parceria entre os três atores institucionais mencionados, é importante destacar a ação de coletivos de gestão com especial referência aos conselhos gestores das UC, ao Conselho da Biosfera da Amazônia Central e ao Conselho do Mosaico do Baixo Rio Negro. Além disso, os financiadores, especialmente o Programa ARPA garantiram a continuidade das ações de gestão destes espaços protegidos.

Em contraste, no Parest Setor Norte a atuação compartilhada entre a entidade parceira de gestão (FVA), a chefia da unidade e lideranças locais não foi tão coesa e consistente ao longo do tempo. As demandas sociais pouco estruturadas a partir das representações comunitárias e o direcionamento para outras ações da gestão por parte do órgão gestor contribuíram para que a revisão de limites e categorias não se estabelecesse como uma ação de gestão prioritária no Parest Setor Norte. 
A discussão da revisão dos limites do Parest Setor Norte acabou voltando ao cenário da geopolítica regional a partir de um evento não conectado diretamente com a conservação que foi a construção de uma cidade universitária. As entidades ambientalistas envolvidas na conservação do rio Negro e representadas no Conselho do CERBAC visualizaram, com este evento, uma oportunidade para avançar nas negociações, tanto internamente junto ao órgão gestor quanto localmente junto aos comunitários residentes no Parest Setor Norte. No entanto, um recente contexto político desfavorável e conturbado na política ambiental do estado do Amazonas, parece ter sido a principal causa do atraso na conclusão do processo de revisão de limites e categoria no Parest Setor Norte.

As análises e narrativas históricas apresentadas neste estudo demonstram que as causas subjacentes aos eventos PADDD tanto podem ser complexas, como podem se estabelecer por meio de uma dinâmica rede de atores. Tal complexidade não pode ser devidamente caracterizada por intermédio de análises genéricas e superficiais, demandando estudos mais pormenorizados. Deste modo, estudos mais regionalizados podem auxiliar no entendimento dos processos e consequências que os eventos de PADDD podem acarretar para a gestão de unidades de conservação na Amazônia.

\subsection{A REVISÃO DE LIMITES E CATEGORIAS PODE SER BENÉFICA PARA A GESTÃO DAS UC?}

Eventos recentes de revisão de limites e categorias têm colocado em pauta a fragilidade institucional de áreas protegidas frente às demandas que conflitam com os objetivos de conservação (MARQUES; PERES, 2015). De fato, eventos desta natureza já afetaram 41 UC na Amazônia brasileira resultando na perda de 88.341,67 hectares (PACK et al., 2016). Esta área representa uma porção pequena do sistema de UC na Amazônia, mas se estes eventos se tornarem muito frequentes, podem se constituir em uma séria ameaça à governança do sistema de áreas protegidas da Amazônia brasileira. De fato, já existem processos em andamento de revisão de limites e categorias de 23 UC na Amazônia brasileira que podem resultar na perda de mais de 191.448 hectares (PACK et al., 2016).

É importante salientar, no entanto, que reconfigurações de limites e categorias de áreas protegidas podem representar oportunidades de aperfeiçoamento da gestão local e de correção de eventuais equívocos e injustiças no processo de criação destas áreas protegidas. A revisão de limites de áreas protegidas com aumento de tamanho também pode ser uma estratégia com bom 
custo-benefício para ampliar o sistema oficial de conservação (NAUGHTONTREVES et al., 2006; FULLER et al., 2010).

As propostas de revisão de categorias de UC podem contribuir para uma composição de áreas protegidas mais bem adaptadas regionalmente, tal como demonstrado nos casos de reclassificações da Estação Ecológica Mamirauá em uma Reserva de Desenvolvimento Sustentável (QUEIROZ, 2005) e da Estação Ecológica de Anavilhanas em um parque nacional (ICMBIO, 2017). Este último caso é emblemático, uma vez que a categoria de estação ecológica se mostrou inadequada, pois a UC sempre foi muito utilizada para a prática do turismo ecológico e como hidrovia. Assim, a recategorização desta unidade para parque nacional, mesmo implicando em uma diminuição na restrição legal (downgrading), facilitou os processos de manejo desta área protegida mais adaptada às demandas de conservação regional (ICMBIO, 2017).

É de fundamental relevância, portanto, incorporar o dinamismo social, econômico e político local no processo de se planejar a criação e manutenção de redes de áreas protegidas (SYMES et al., 2016). Um componente de destaque deste dinamismo se refere aos conflitos fundiários que surgem devido à incompatibilidade de destinação de áreas a diferentes grupos de interesse e que afetam a estabilidade espacial do conjunto de áreas protegidas.

Inúmeras comunidades residem dentro dos limites de UC de proteção integral na Amazônia e em outros biomas. Esta situação é irregular, segundo a legislação ambiental, tanto no âmbito federal quanto estadual. A legislação ambiental, notadamente no capítulo IX do SNUC (BRASIL, 2000), define que a solução para esta irregularidade é a justa indenização e adequada realocação destes moradores. No entanto, processos indenizatórios e de realocação de famílias raramente são conduzidos pelos órgãos gestores por falta de recursos financeiros ou vontade política. Mesmo nos casos onde esta solução foi implementada, observa-se vários casos de conflitos entre os órgãos gestores e as comunidades locais (BARRETTO FILHO, 1999).

Apesar de uma análise detalhada das consequências da revisão de limites e categoria do Parest Setor Sul estar além do escopo deste estudo, foi observado que este processo parece ter implicado em alguns benefícios para a gestão da UC. O diálogo com as comunidades foi facilitado, uma vez que existe o amparo legal para a promoção de atividades geradoras de renda no contexto da RDS Puranga Conquista. Além disso, as comunidades passaram a ter uma preocupação maior com a gestão dos recursos naturais da região, inclusive do setor que permaneceu com status de parque estadual. De fato, os Conselhos Gestores das duas UC 
têm trabalhado numa perspectiva de gestão compartilhada entre a RDS Puranga Conquista e o Parest Setor Sul.

O aspecto negativo da revisão de limites foi a redução da área do Parest Setor Sul. Considerando-se a área definida na primeira revisão de limites de 2001, houve uma significativa redução de cerca de $50 \%$ da área do parque. Ainda é necessário avaliar as implicações desta perda de área em termos de proteção da biodiversidade e ecossistemas regionais. Além disso, o fato do Parest Setor Sul estar mais próximo de Manaus pode resultar em maior pressão sobre os recursos naturais da RDS Puranga Conquista e Parest Setor Sul, um aspecto que merece uma investigação mais detalhada.

No caso do Parest Setor Norte é previsto um processo de revisão de limites com um ganho substancial de área para a unidade (compare Figura 3C e 3D). No entanto, como o processo legal ainda não se concretizou, não é possível antever os potenciais benefícios que esta revisão de limites venha a trazer para a proteção e o uso dos recursos naturais da região.

A revisão de limites e categorias pode ser uma estratégia válida de resolução de parte dos conflitos fundiários de populações que residem em unidades de proteção integral, especialmente naquelas criadas antes da Lei do Sistema Nacional de Unidades de Conservação. Esta estratégia, no entanto, não deve ser banalizada com risco de fragilizar a governança das UC com prejuízos para a biodiversidade. As propostas de revisão de limites e categorias devem ser analisadas caso a caso e baseadas em análises técnico-científicas robustas e consistentes. Estas propostas devem constar dos planos de manejo ou de gestão das UC em análise e devem fazer parte contínua do debate junto aos conselhos gestores. Além disso, estas propostas devem ser amplamente negociadas entre as comunidades locais, parceiros de gestão, órgãos gestores e a classe política para se evitar prejuízos na proteção da biodiversidade regional. Esperamos que os resultados obtidos na síntese historiográfica apresentada neste estudo sejam úteis no debate de revisão de limites e categorias de UC em outros contextos na Amazônia.

\section{REFERÊNCIAS}

AMAZONAS. Lei Complementar $\mathbf{N}^{\circ}$ 53, 05 junho 2007. Regulamenta o inciso V do artigo 230 e o $\int 1^{\circ}$ do artigo 231 da Constituição Estadual, institui o Sistema Estadual de Unidades de Conservação - SEUC, dispondo sobre infrações e penalidades e estabelecendo outras providências. Manaus: Assembleia Legislativa, 2007. Disponível em: $\quad$ http://conservacao.mpambiental.org/wp-content/uploads/2015/05/Lei_ Complementar_53_2007_institui_SEUC.pdf. Acesso em: 25 jul. 2019. 
AMAZONAS. Plano de gestão do Parque Estadual do Rio Negro - Setor Norte. Manaus: Secretaria de Meio Ambiente e Desenvolvimento Sustentável do Estado do Amazonas (SDS)/Fundação Vitória Amazônica (FVA), 2008.

AMAZONAS. Plano de gestão do Parque Estadual do Rio Negro - Setor Sul. Manaus: Secretaria de Meio Ambiente e Desenvolvimento Sustentável do Estado do Amazonas (SDS)/Instituto de Pesquisas Ecológicas (IPE), 2010.

BARRETTO FILHO, H. T. Notas para a história de um artefato cultural: o Parque Nacional do Jaú. Terras das Águas - Revista de Estudos Amazônicos, Brasília, v. 1, p. $53-76,1999$.

BECKER, B. Geopolítica da Amazônia. Estudos Avançados, São Paulo, v. 19, p. 71-86, 2005.

BERNARD, E.; PENNA, L. A.; ARAÚJO, E. Downgrading, Downsizing, Degazettement, and Reclassification of Protected Areas in Brazil. Conservation Biology, Washington, v. 28, p. 939-950, 2014.

BRASIL. Lei no 9.985, 18 jul. 2000. Regulamenta o art. 225, \ 1º, incisos I, II, III e VII da Constituição Federal, institui o Sistema Nacional de Unidades de Conservação da Natureza e dá outras providências. Diário Oficial da União, Brasília, DF, 19 jul. 2000. Disponível em: http://www.planalto.gov.br/ccivil_03/leis/19985.htm. Acesso em: 25 jul. 2019.

FULLER, R. A.; MCDONALD-MADDEN, E.; WILSON, K. A.; CARWARDINE, J.; GRANTHAM, H. S.; WATSON, J.; KLEIN, C. J.; GREEN, D. C.; POSSINGHAM, H. P. Replacing underperforming protected areas achieves better conservation outcomes. Nature, New York, v. 466, p. 365-367, 2010.

FVA. Fundação Vitória Amazônia, Manaus, sem data. Disponível em: https://www. fva.org.br/home/. Acesso em: 26 jul. 2019.

ICMBIO. Plano de Manejo do Parque Nacional de Anavilhanas. Novo Airão: Instituto Chico Mendes de Conservação da Biodiversidade (ICMBIO)/Instituto de Desenvolvimento Sustentável do Amazonas (IDESAM), 2017.

JUFFE-BIGNOLI, D.; BURGESS, N. D.; BINGHAM, H.; BELLE, E. M. S.; DE LIMA, M. G.; DEGUIGNET, M.; BERTZKY, B.; MILAM, A. N.; MARTINEZ-LOPEZ, J.; LEWIS, E.; EASSOM, A.; WICANDER, S.; GELDMANN, J.; VAN SOESBERGEN, A.; ARNELL, A. P.; O’CONNOR, B.; PARK, S.; SHI, Y. N.; DANKS, F. S.; MACSHARRY, B.; KINGSTON, N. Protected Planet Report 2014. UNEP-WCMC: Cambridge, UK, 2014.

LITTLE, P. Megaproyectos en la Amazonía: un análisis geopolítico y socioambiental con propuestas de mejor gobierno para la Amazonía. Lima: RAMA, ARA, DAR, 2013.

MARQUES, A. A.; PERES, C. A. Pervasive legal threats to protected areas in Brazil. Oryx, Cambrigde, v. 49, p. 25-29, 2015.

MASCIA, M. B.; PAILLER, S. Protected area downgrading, downsizing, and degazettement (PADDD) and its conservation implications. Conservation Letters, v. 4, p. 9-20, 2011. 
MASCIA, M. A.; PAILLER, S.; KRITHIVASAN, R.; ROSHCHANKA, V.; BURNS, D.; MLOTHA, M. J.; MURRAY, D. R.; PENG N. Protected area downgrading, downsizing, and degazettement (PADDD) in Africa, Asia, and Latin America and the Caribbean, 1900-2010. Biological Conservation, New York, v. 169, p. 355-361, 2014.

NAUGHTON-TREVES, L.; ALVAREZ-BERRÍOS, N.; BRANDON, K.; BRUNER, A.; HOLLAND, M. B.; PONCE, C.; SAENZ, M.; SUAREZ, L.; TREVES, A. 2006. Expanding protected areas and incorporating human resource use: a study of 15 forest parks in Ecuador and Peru. Sustainability: Science, Practice, \& Policy, v. 2, p. 32 44, 2006.

PACK, S.; FERREIRA, M. N.; KRITHIVASAN, R.; MURROWD, J.; BERNARD, E.; MASCIA, M. B. Protected area downgrading, downsizing, and degazettement (PADDD) in the Amazon. Biological Conservation, v. 197, p. 32-39, 2016.

PENNA, L. A. Redução, desclassificação e reclassificação de unidades de conservação no Brasil. 2013. Trabalho de Conclusão de Curso (Graduação em Biologia) - Centro de Biociências, Universidade Federal de Pernambuco, Recife, 2013.

QUEIROZ, H. L. A reserva de desenvolvimento sustentável Mamirauá. Estudos Avançados, São Paulo, v. 19, p. 183-203, 2005.

SYMES, W.; RAO, M.; MASCIA, M. B.; CARRASCO, L. R. Why do we lose protected areas? Factors influencing protected area downgrading, downsizing and degazettement in the tropics and subtropics. Global Change Biology, v. 22, p. 656-665, 2016.

UNIDADES DE CONSERVAÇÃO NO BRASIL. Instituto Socioambiental, Brasília, sem data. Painel de dados. Disponível em: https://uc.socioambiental.org/pt-br/ paineldedados. Acesso em: 26 jul. 2019.

UNIDADES DE CONSERVAÇÃO NO BRASIL. Instituto Socioambiental, Brasília, sem data. Sistemas de unidades de conservação. Disponível em: https:/ / uc.socioambiental. org/prote\%C3\%A7\%C3\%A3o-integral/parques. Acesso em: 26 jul. 2019. 
